\title{
A rare but life-threatening complication in liver transplant recipients
}

\author{
Joanna Raszeja-Wyszomirska ${ }^{1}$, Michał P. Wasilewicz ${ }^{1}$, Monika Szydłowska-Jakimiuk ${ }^{1}$, Ireneusz Grzelak², \\ Wojciech Figiel ${ }^{2}$, Wojciech Sachs ${ }^{3}$, Grzegorz Niewiński ${ }^{4}$, Beata Gierej ${ }^{5}$, Bogna Ziarkiewicz-Wróblewska ${ }^{5}$, \\ Marek Krawczyk ${ }^{2}$
}

${ }^{1}$ Liver and Internal Medicine Unit, Department of General, Transplant, and Liver Surgery, Medical University of Warsaw, Warsaw, Poland

2Department of General, Transplant, and Liver Surgery, Medical University of Warsaw, Warsaw, Poland

${ }^{3}$ Department of Haematology and Oncology, Medical University of Warsaw, Warsaw, Poland

${ }^{4}$ II Department of Anaesthesiology and Intensive Care, Medical University of Warsaw, Warsaw, Poland

${ }^{5}$ Department of Pathology, Medical University of Warsaw, Warsaw, Poland

Prz Gastroenterol 2016; 11 (1): 62-64

DOI: $10.5114 /$ pg.2015.52562

\begin{abstract}
Address for correspondence: Joanna Raszeja-Wyszomirska MD, Liver and Internal Medicine Unit, Department of General, Transplant, and Liver Surgery, Medical University of Warsaw, 1 A Banacha St, 02-097 Warsaw, Poland, phone: +48 225991662 , fax: +48 225991663 , e-mail: joanna.wyszomirska@wum.edu.pl
\end{abstract}

Graft versus host disease (GvHD) occurs in as little as $1-2 \%$ of cases after liver transplantation (LT), but is probably under-diagnosed and under-reported. Skin rash, diarrhoea, and/or fever are early symptoms of GvHD, and the most common causes of death are sepsis or gastrointestinal bleeding as a result of bone-marrow involvement. The delay in diagnosis as well as lack of standard treatment contributes to the high lethality of GVHD.

A 45-year-old patient, six weeks after liver transplantation because of primary sclerosing cholangitis (PSC), was admitted to the hospital due to watery diarrhoea. The patient presented dehydration with more than 20 watery, periodically bloody stools with spastic mid-abdominal pain, slight elevation of body temperature, and merging redness rush on feet and hands. C-reactive protein (CRP) was reached more than $300 \mathrm{mg} / \mathrm{dl}$. All stool bacterial cultures were negative, including Clostridium difficile toxaemia, cytomegalovirus, and Epstein-Barr virus infections. Because of the patient's history of ulcerative colitis, rectosigmoidoscopy was performed showing moderate exacerbation of the disease, confirmed by the result of a colonic biopsy. Unfortunately, the symptomatic treatment was ineffective, with a persistent high number of stools per day, with elevation of CRP, fibrinogen and dyselectrolytaemia, anaemia, and decreasing counts of leucocytes and platelets. His maculopapular rush developed occupying also the abdomen, chest, and back (Figures 1 A, B). Computer tomography revealed inflammation of both small and large intestines with as- cites (Figure 2). The patient was treated with tacrolimus (drug blood levels of 8-12 ng/ml), basiliximab (20 mg i.v. in day 0 and 4), and methylprednisolone $500 \mathrm{mg}$ IV q.d. for 3 days, subsequently with oral prednisolone and budesonide, antibiotics, and antifungal drugs as well as with intravenous colloids, crystalloids, human albumin, ions, and nutritional support. However, general vasculitis and multiorgan failure, secondary to fungal sepsis, developed and the patient died 30 days after onset.

A 54-year-old male patient, 1 month after liver transplantation due to HCV cirrhosis, was admitted to the hospital with a 3-day history of fever and dysuric symptoms. Chinolon was administered ambulatorily, but his fever grew growing, with very high CRP, procalcitonin, and pancytopaenia. His condition deteriorated in a short time, with skin rash on the chest and abdomen, and with severe diarrhoea. Bone marrow and skin biopsies were taken as well as chimerism of host- and donor-circulating lymphocytes was assessed, with confirmation of acute GvHD. The immune suppression regimen with calcineurin inhibitor (CNI) tacrolimus (drug blood levels of 8-12 $\mathrm{ng} / \mathrm{ml}$ ), basiliximab (20 mg i.v. in day 0 and 4), methylprednisolone $500 \mathrm{mg}$ i.v. q.d. for 3 days, and mTOR inhibitor everolimus was introduced, as well as wide-spectrum antibiotics and antifungal drug, but the patient died due to multi-organ failure followed by massive gastrointestinal bleeding.

Fever, skin rash, diarrhoea, severe bone marrow suppression, and gastrointestinal bleeding beginning 


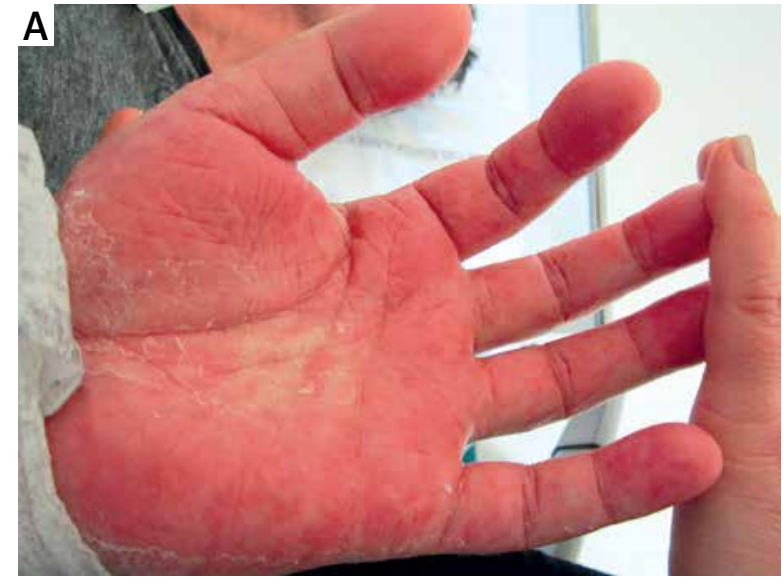

Figure 1 A, B. Maculopapular skin rush

2 to 6 weeks after liver transplantation (LT) are clinical manifestations of acute GvHD. Graft versus host disease following liver transplantation is an uncommon but fatal complication that poses a major diagnostic and therapeutic challenge. Research has shown that GvHD involves activation of donor T lymphocytes by antigen-presenting cells (APCs), causing an alloreactive T-cell response to recipient tissues mediated by cytotoxic T-cells and inflammatory cytokines [1], such as tumour necrosis factor- $\alpha$ (TNF- $\alpha$ ), interleukins $1,2,6$, and 10, and interferon $\gamma$ [2]. Of these cytokines, TNF- $\alpha$ is recognised as the key inflammatory cytokine involved in the pathogenesis of GVHD, which can activate APCs, recruit effector cells, and cause tissue damage [2, 3]. Most cases of GVHD after LT are diagnosed by typical clinical manifestations, but laboratory examinations can help with the diagnosis: (1) biopsy of the skin rash; (2) detection of donor peripheral blood leucocytic chimerism; (3) microsatellite phenotype; (4) detection of donor HLA types in the peripheral blood, mucous membrane, or skin by PCR; and (5) detection of donor lymphocytes using immunohistochemistry on the skin rash [4].

Although the liver is recognised as an immunologically privileged organ, acute graft versus host disease may occur in $1-2 \%$ of patients after $L T$, with very high mortality (> 85\%) [5-7]. Due to the low incidence of the disease and difficulty in evaluating the efficacy of treatment modalities, the therapeutic schedule for GvHD after LT has not yet been standardised. Most treatment methods are derived from those experiences of GvHD management following bone marrow transplantation (HSCT). There are two main therapeutic strategies: to increase the immunosuppression or to withdraw the immunosuppressants to control GVHD as suggested by Chinnakotla et al. [8]. Unfortunately, the complication of the former strategy is usually fatal sepsis, and the latter option also does not allow control of the progression of GvHD.
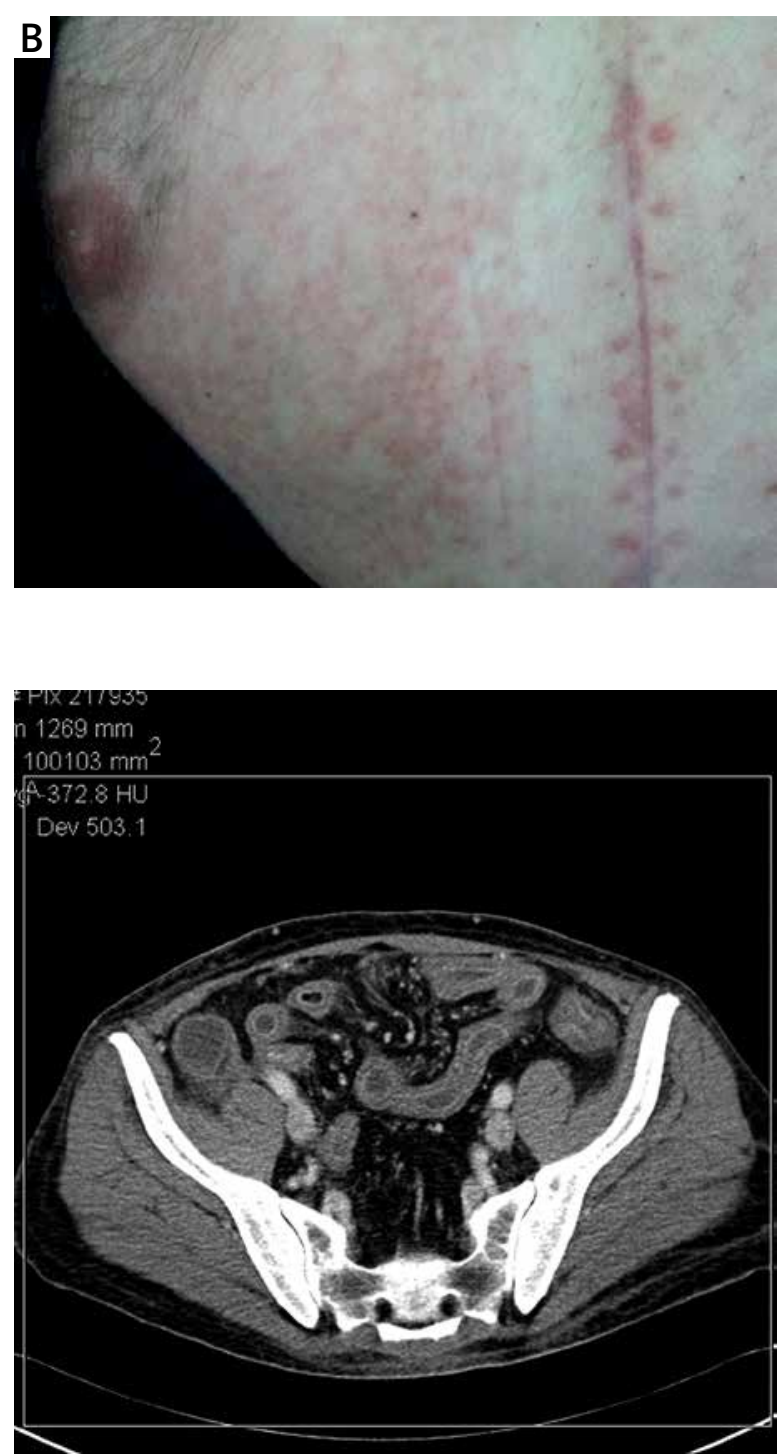

Figure 2. Computed tomography scan of abdomen with swelling of the intestine

In the above-mentioned cases the diagnosis of aGvHD was supported by rash skin biopsy, after the exclusion of other possible reasons for diarrhoea and rash, as well as with detection of donor peripheral blood leucocytic chimerism. Our therapy included high-dose corticosteroids because it was considered an important part of first-line treatment for acute GvHD after LT [5-7]. The use of corticosteroids for the treatment of GVHD after LT is derived from the HSCT experience: corticosteroids resolve symptoms in many patients. The mechanism by which glucocorticoids ameliorate acute GVHD is not completely clear, but it is probably related primarily to the suppression of lymphocytic activity. However, GvHD after LT is less responsive to corticosteroids than GVHD after stem cell transplantation. Based on the literature, both major strategies 
- augmentation or withdrawal of immunosuppressant therapy - seem to be ineffective in GvHD treatment [9], so we decided to apply a novel approach with interleukin 2-receptor antibody basiliximab. With the other supportive care we saw an improvement in the patient's general condition; however, fungal sepsis developed with the need of immunosuppression reduction. The described patient died after 30 days of the therapy, which is a longer time than previously reported [9]. The reduction of immunosuppression, caused by sepsis, promoted the progression of GvHD to general vasculitis and multi-organ failure, supporting the thesis of the necessity for powerful immunosuppression in the therapy, with modern additional care.

Although GVHD is a rare complication after LT, it can lead to significant mortality, deepen by the delay of diagnosis and the lack of efficacious treatment. Clinical features represent an important tool for early diagnosis. Further research is needed to provide new therapeutic agents for treating this condition effectively.

\section{Conflict of interest}

The authors declare no conflict of interest.

\section{References}

1. Mawad R, Hsieh A, Damon L. Graft-versus-host disease presenting with pancytopenia after en bloc multiorgan transplantation: case report and literature review. Transplant Proc 2009; 41: 4431-3.

2. Ferrara JL, Levine JE, Reddy P, Holler E. Graft-versus-host disease. Lancet 2009; 373: 1550-61.

3. Piton G, Larosa F, Minello A, et al. Infliximab treatment for steroid-refractory acute graft-versus-host disease after orthotopic liver transplantation: a case report. Liver Transpl 2009; 15: 682-5.

4. Lu Y, Wu LQ, Zhang BY, Cao JY. Graft-versus-host disease after liver transplantation: successful treatment of a case. Transplant Proc 2008; 40: 3784-6.

5. Smith DM, Agura E, Netto G, et al. Liver transplant-associated graft-versus-host disease. Transplantation 2003; 75: 118-26.

6. Taylor AL, Gibbs P, Bradley JA. Acute graft versus host disease following liver transplantation: the enemy within. Am J Transplant 2004; 4: 466-74.

7. Taylor AL, Gibbs P, Sudhindran S, et al. Monitoring systemic donor lymphocyte macrochimerism to aid the diagnosis of graftversus-host disease after liver transplantation. Transplantation 2004; 77: 441-6.

8. Chinnakotla S, Smith DM, Domiati-Saad R, et al. Acute graftversus-host disease after liver transplantation: role of withdrawal of immunosuppression in therapeutic management. Liver Transpl 2007; 13: 157-61.

9. Xiao-Bo C, Jie Y, Ming-Qing X, et al. Unsuccessful treatment of four patients with acute graft-vs-host disease after liver transplantation. World J Gastroenterol 2012; 18: 84-9.

Received: 6.01 .2015

Accepted: 11.02 .2015 